\title{
Carta al editor respecto del suplemento 1, 2020 de la pandemia por SARS-CoV-2
}

\section{Letter to the editor regarding Supplement 1, 2020 on the SARS-CoV-2 pandemic}

Arturo Loredo-Abdalá, ${ }^{1}$ César Alejandro Galván-Díaz, ${ }^{2}$ Nancy Evelyn Aguilar-Gómez, ${ }^{3}$ Linsay Krystel CervantesPérez, ${ }^{4}$ Pablo Alfredo Ojeda-Pérez, ${ }^{5}$ Abigail Casas-Muñoz ${ }^{6}$

Hemos leído con satisfacción la publicación en Acta Pediátrica de México del Volumen 41, Suplemento 1, 2020 que trata sobre la pandemia por SARS-CoV-2 que genera COVID-19 y que mundialmente está afectando al ser humano. ${ }^{1}$ En éste, se hace una revisión exhaustiva de los acontecimientos más importantes de la pandemia por el virus SARS-CoV-2 y la enfermedad que origina COVID-19. De esta manera, el personal médico y paramédico del Instituto Nacional de Pediatría así como los lectores de Acta Pediátrica de México podrán estar enterados del conocimiento que a nivel mundial, actualmente se tiene sobre la epidemiología, fisiopatología de la infección, del cuadro clínico en niños, los procedimientos de laboratorio y gabinete requeridos para establecer el diagnóstico, la estrategia desarrollada para su atención de emergencia, el estado actual para el desarrollo de una vacuna y, lo más importante, por el momento, se presentan las medidas requeridas universalmente para que la población humana no adquiera la infección. ${ }^{2}$

Dos aspectos son relevantes en la realización y presentación de este Suplemento:

a. Cada artículo, generalmente requirió desde su presentación a su aceptación editorial, un promedio de 3 a 4 semanas (mayo-junio) y el Suplemento salió publicado dos o tres semanas mas tarde (mes junio). Ello habla de la disposición que los directivos del INP y de Acta Pediátrica de México tuvieron para realizar, en tiempo récord, esta publicación.

b. Por otra parte, toda la información vertida en este Suplemento se encuentra sustentada en bibliografía internacional seleccionada con rigor científico.

\footnotetext{
${ }^{1}$ Médico pediatra, internista, especialista en la atención del niño maltratado, coordinador de la Coordinación de Estudios Avanzados sobre Maltrato Infantil-Prevención.

${ }^{2}$ Médico pediatra oncólogo, alumno del curso de posgrado de maltrato infantil, adscrito al servicio de Oncología.

${ }^{3}$ Médica pediatra infectóloga, alumna del curso de posgrado de maltrato infantil, adscrita al Departamento de Infectología.

${ }^{4}$ Médica pasante de Servicio Social, Universidad Nacional Autónoma de México, en la Coordinación de Estudios Avanzados sobre Maltrato InfantilPrevención.

${ }^{5}$ Médico pasante de Servicio Social de la Universidad Nacional Autónoma de México, en la Coordinación de Estudios Avanzados sobre Maltrato InfantilPrevención

${ }^{6}$ Coordinación de Estudios Avanzados sobre Maltrato Infantil-Prevención, Instituto Nacional de Pediatría
}

Recibido: 2 de marzo 2020

Aceptado: 3 de septiembre 2020

Correspondencia Abigail Casas-Muñoz abycas.md@gmail.com

Este artículo debe citarse como Casas-Muñoz A, Loredo-Abdalá A, Galván-Díaz CA, Aguilar-Gómez NE, Cervantes-Pérez LK, Ojeda-Pérez PA. Carta al editor con respecto del suplemento 1, 2020 sobre la pandemia por SARS-CoV-2. Acta Pediatr Méx 2020; 41(5): 235-39. 
En un afán por colaborar en el esfuerzo académico y editorial realizado, y tomando en consideración que la pandemia en México no está ni remotamente controlada, nos hemos dado a la tarea de hacer las siguientes consideraciones, para evitar que las niñas, niños y adolescentes que aparentemente están sanos y los que sufren alguna comorbilidad, no se infecten. De esta manera, intentamos apoyar a todos los médicos, a los profesionales paramédicos y a los lectores de Acta Pediátrica de México para que extiendan esta información y la apliquen al realizar su práctica profesional.

Como ya se mencionó, una faceta que no fue considerada totalmente en el Suplemento fue no atender integralmente a la población infantojuvenil aparentemente sana o aquella que cursa con alguna comorbilidad para que no adquirieran la infección.

Ello, nos dio la pauta para insistir al grupo médico y paramédico sobre la importancia que tienen las estrategias de prevención en numerosos problemas que afectan la salud y las vidas de las niñas, niños y adolescentes, como son los accidentes, las infecciones virales o bacterianas por no lavarse las manos, las vacunas, etc. y en este momento, la pandemia generada por el virus SARS-CoV-2 no debe ser la excepción. ${ }^{3}$

Desarrollar otras estrategias preventivas que se suman a las mundialmente aceptadas (lavado de manos, aseo bucal frecuente, el manejo correcto de la tos o el estornudo, mantener la sana distancia, el uso constante de cubre bocas) varía si se está frente una población infanto-juvenil con alguna comorbilidad o ante una población aparentemente sana. ${ }^{3}$

Dentro del primer grupo tenemos a dos poblaciones:

\section{a. Niñas, niños 0 adolescentes con alguna comorbilidad, pero sin COVID-19}

En general, la mayoría de los pacientes con cáncer, algún padecimiento hematológico, otros que provocan inmunosupresión o una enfermedad crónica, precisan seguimiento médico constante. La continuidad de los diversos protocolos dependerá del momento clínico en que se encuentre cada paciente. Para este grupo, se incrementarán las medidas de diagnóstico del contagio por SARS-CoV-2 y aún en los asintomáticos, como una medida aparentemente estándar, antes de iniciar una fase de riesgo en su tratamiento de su enfermedad principal (por ejemplo, inducción a la remisión en un paciente con leucemia) y evidentemente, se aplicarán todas las estrategias preventivas. Obviamente, este grupo de menores tendrán que ser sometidos rigurosamente a las estrategias preventivas contra la COVID-19, universalmente aceptadas y en todos sus contactos. ${ }^{4-5}$

Por otro lado, una insistencia en lo que corresponde al manejo de la salud mental de estos niños. Este deberá ser más intenso, en virtud de que si están hospitalizados, sus padres no permanecen mucho tiempo con ellos. Por lo tanto, esta situación seguramente les generará ansiedad y tristeza. Un apoyo podrá ser una mayor comunicación telefónica o la implementando de video conferencias.

\section{b. Niñas, niños y adolescentes sanos y sin comorbilidad}

En el campo de la prevención, haremos algunas propuestas que los padres, familiares, médicos y el personal paramédico podrán seguir: 


\section{Consideraciones básicas}

\section{Aseo personal}

Lavado frecuente de las manos con agua y jabón durante 20-30 segundos o alcohol gel al 60\%, por al menos 20 segundos asegurando cubrir toda la superficie de las manos; baño diario si es posible y aseo bucal $4-5$ veces al día. ${ }^{6-7}$

\section{Inmunizaciones}

a) La pandemia no debe impedir que se cubra en su totalidad el Esquema Nacional de Vacunación, de acuerdo con su edad. Idealmente, este debe ser cubierto en su totalidad. Si no es así, a medida que la evolución de la epidemia de COVID-19 lo permita, las inmunizaciones se reanudarán y recuperarán, recomendando entonces priorizando a los lactantes menores, pacientes con enfermedades crónicas e inmunodeprimidos, los que conviven con personas (niños o adultos) de mayor riesgo y a las embarazadas, siguiendo en todo caso, las instrucciones de los servicios regionales de salud..$^{8-9}$

Para ello, se deben vencer los siguientes obstáculos:

b) Que los padres no tengan miedo de ir al hospital, a un centro de salud o a un consultorio privado porque tienen la idea de que ahí se pueden infectar sus hijos. Previamente, deben precisar que en dicho centro, exista un área exclusiva para la atención de pacientes con sospecha de COVID-19 (plan de biocontingencia) y otro para la aplicación de las vacunas en niños aparentemente sanos. ${ }^{10}$
C) Si en la familia hay personas con COVID-19 el niño se debe mantener bajo una estrecha vigilancia durante 14 días. Si se precisa que el menor no tiene infección por el virus SARS-CoV-2 después de ese período, se puede vacunar de acuerdo con su edad.

d) Si el niño tiene en ese momento un padecimiento agudo (diarrea, gripe, crisis de alergia, etc.) los familiares se pueden esperar unos días hasta la solución de dicho problema.

e) Los familiares deben hacer caso omiso, a señalamientos que un grupo de personas hacen e insisten de que las vacunas no sirven para proteger a los niños, argumentando que solamente son útiles para enriquecer a las Farmacéuticas. ${ }^{11}$

\section{Alimentación}

La alimentación de los menores debe ser completa, suficiente, equilibrada e higiénica. Así mismo, se debe recordar que la actividad física de los niños se ha disminuido considerablemente; por lo tanto, la vigilancia de estas dos situaciones es fundamental para evitar que adquieran, carencia de algún nutrimento específico (complejo B, vitaminas A, C, D) desnutrición de grado variable, sobrepeso u obesidad. ${ }^{12}$

\section{Vigilancia de la Salud Mental y prevención de violencia}

En el transcurso de esta pandemia, la atención de la salud mental de las niñas, niños y adolescentes sanos es muy importante, ya que se desarrollan varios eventos que están cambiando sustancialmente su vida y la de sus padres. El mantenerse en la casa 
las 24 h del día; la convivencia continua con los hermanos y con sus padres, el no poder salir a la calle o no asistir a la escuela, el no estar con los compañeros del colegio son para los niños una novedad poco agradable. Si a ello le agregamos la ansiedad, angustia, depresión de los padres, la condición familiar se puede tornar caótica. ${ }^{13}$

Considerar y agregar a lo anterior, el tipo de familia; esta puede ser, entre otras formas monoparental, disfuncional, con adicciones lícitas e ilícitas, conductas disociales, etc. Ello, seguramente causará en los menores, estrés, angustia, ansiedad y/o alteración del sueño, principalmente. ${ }^{14}$ Las posibilidades de que en este escenario se desarrolle con mayor frecuencia violencia familiar y en algunos casos, maltrato infantil, han sido notificados. ${ }^{15}$ Para su prevención, los padres y sus familiares deben fortalecer en la niña, niño o adolescente su resiliencia, espiritualidad, valores, etc. mediante la implementación de diversas actividades recreativas (dibujo, uso de pinturas y plastilina, cantos y bailes).

El médico general o el pediatra deben estar atentos a la aparición de algunas anormalidades como son: ansiedad, depresión, miedo, estrés postraumático, etc. con mayor severidad, podrían requerir la intervención del especialista en salud mental.

En los niños escolares y adolescentes la educación a distancia, al seguir las actividades escolares por 5-6 h en la tableta electrónica o en la computadora, es muy probable que cause resequedad de la córnea y/o agrave alteraciones en la visión (miopía, astigmatismo), así como ocasionar cefalea y malestar general que se podrían confundir con situaciones más graves. El diagnóstico temprano de dichos problemas oculares evitará el agravamiento del problema, así como falla en el rendimiento escolar. ${ }^{16,17}$

\section{Educación}

La selección del esquema educativo que deben seguir los niños sanos, inmersos en la "cuarentena" dependerá de su edad, condición socioeconómica y el tipo de escuela que le convenga. ${ }^{18}$

\section{Actividad física}

Los padres o los familiares responsables de los niños aparentemente sanos deben considerar el establecimiento de una rutina de actividad física que permita no solamente su distracción con dicha actividad, si no también deberá permitir ejercitar a los niños. El programa seleccionado e implementado por los padres dependerá del espacio disponible en la casa, edificio, patio o la posible asistencia controlada a un jardín o parque. ${ }^{19}$

\section{Juegos y recreación}

Se debe favorecer y estimular que el niño juegue constantemente, dibuje o use plastilina u otros juguetes creativos y recreativos. Estas actividades le distraerán y se disminuirá el aburrimiento. Es muy importante limitar el uso excesivo de dispositivos electrónicos. El desarrollar éstas y otras actividades similares con adultos y/o hermanos, son una buena manera para fortalecer el vínculo con ellos. ${ }^{20}$

Es nuestro deseo que la implementación de las estrategias señaladas ante los diversos panoramas que pueden vivir las niñas, niños y adolescentes durante la Pandemia, permitan que la salud física y emocional de las personas de este grupo etario salga adelante con un mínimo daño.

\section{REFERENCIAS}

\footnotetext{
1. Serrano Sierra A. Prólogo. Acta Pediatr Mex. 2020; 41(Supl 1): 1-2.

2. Saltigeral Simental P, León-Lara X. Virus SARS-CoV-2 ¿Qué se sabe al momento? Acta Pediátr México. 2020; 41 (4S1):3-7.
} 
Casas-Muñoz A, et al. Carta al editor con respecto del suplemento 1, 2020

3. Liu J, et al. Community Transmission of Severe Acute Respiratory Syndrome Coronavirus 2, Shenzhen, China, 2020. Emerg Infect Dis. 2020; 26: 1320-3.

4. Baruchel A, et al. COVID-19 and acute lymphoblastic leukemias of children and adolescents: First recommendations of the Leukemia committee of the French Society for the fight against Cancers and Leukemias in children and adolescents (SFCE). Bull Cancer. 2020 Apr;

5. Balduzzi $A$, et al. Lessons after the early management of the COVID-19 Outbreak in a Pediatric Transplant and Hemato-Oncology Center Embedded within a COVID-19 Dedicated Hospital in Lombardia, Italy. Estote Parati. (Be Ready.). SSRN Electron J [Internet]. 2020. https://www. ssrn.com/abstract $=3559560$

6. Ghinai I, et al. First known person-to-person transmission of severe acute respiratory syndrome coronavirus 2 (SARSCoV-2) in the USA. Lancet. 2020; 395: 1137-44.

7. Bains V, Bains R. Is oral hygiene as important as hand hygiene during COVID-19 pandemic? Asian Journal of Oral Health and Allied Sciences. 2020; 10: 5.

8. Saxena $S$, et al. Routine vaccination during COVID-19 pandemic response. BMJ. 2020;369:m2392

9. Mcdonald $\mathrm{HI}$, et al. Early impact of the coronavirus disease (COVID-19) pandemic and physical distancing measures on routine childhood vaccinations in England, January to April 2020. Eurosurveillance. 2020; 25 (19): 1-6.

10. Abbas K, et. al. Routine childhood immunization during the COVID-19 pandemic in Africa; a benefit- risk analysis of health benefits versus excess risk of SARS-COV-2 infection. Lancet 2020; S2214-109X (20): 30308-9.
11. GhoshR, et al. Impact of COVID-19 on children: special focus on the psychosocial aspect. Minerva Pediatr 2020; 72: 22635. doi. 10.23736/S0026-4946.20.05887-9)

12. Euro.who.int. 2020 [citado 1 Agosto 2020]. https://www. euro.who.int/en/health-topics/health-emergencies/coronavirus-COVID-19/technical-guidance/food-and-nutritiontips-during-self-quarantine.

13. Duan $L$, et al. An investigation of mental health status of children and adolescents in china during the outbreak of COVID-19. J Affect Disord. 2020; 275: 112-18.

14. Silva Junior F, et al. Impact of COVID-19 pandemic on mental health of young people and adults: a systematic review protocol of observational studies. BMJ Open. 2020; 10 (7): 1-6.

15. Saurabh K, Ranjan S. Compliance and Psychological Impact of Quarantine in Children and Adolescents due to COVID-19 Pandemic. Indian J Pediatr 2020; 87: 532-36.

16. Mufti $M$, et al. Does digital screen exposure cause dry eye? Indian J Clin Anat Physiol. 2019; 6(1):68-72. doi:10.18231/2394-2126.2019.0017

17. Kekunnaya R, Deshmukh A, Badakere A, Sheth J, Bhate M, Kulkarni S. Pivoting to teleconsultation for paediatric ophthalmology and strabismus: Our experience during COVID-19 times. Indian J Ophthalmol. 2020:1387-91.

18. Wang $G$, et al. Mitigate the effects of home confinement on children during the COVID-19 outbreak. Lancet. 2020;395:945-947.

19. Guan $\mathrm{H}$, et al. Promoting healthy movement behaviors among children during the COVID-19 pandemic. Lancet Child Adolesc. 2020; 4 (6): 416-18.

20. WHO. Parenting for lifelong of health COVID-19: 24/7 Parenting Proven parenting tips for all ages in world wide languages. https://www.covid19parenting.com/ 